\title{
Limits on anomalous top-quark gauge couplings from Tevatron and LHC data
}

\author{
M. Fabbrichesi ${ }^{1, \mathrm{a}}$, M. Pinamonti ${ }^{2, \mathrm{~b}}$, A. Tonero ${ }^{3, \mathrm{c}}$ \\ ${ }^{1}$ INFN Trieste, Via Valerio 2, 34127 Trieste, Italy \\ 2 INFN Trieste gruppo collegato di Udine and SISSA, Via Bonomea 265, 34136 Trieste, Italy \\ ${ }^{3}$ ICTP-SAIFR and IFT UNESP, Rua Dr. Bento Teobaldo Ferraz 271, 01140-070 São Paulo, Brazil
}

Received: 22 August 2014 / Accepted: 22 November 2014 / Published online: 13 December 2014

(C) The Author(s) 2014. This article is published with open access at Springerlink.com

\begin{abstract}
We review and update current limits on possible anomalous couplings of the top quark to gauge bosons. We consider data from top quark decay (as encoded in the $W$ boson helicity fractions) and single-top production (in the $t$-, $s$ - and $W t$-channels). We find improved limits with respect to previous results (in most cases of almost one order of magnitude) and extend the analysis to include four-quark operators. We find that new electroweak physics is constrained to live above an energy scale between $430 \mathrm{GeV}$ and $3.2 \mathrm{TeV}$ (depending on the form of its contribution). For comparison, strongly interacting new physics is bounded by scales higher than 1.3 or $1.5 \mathrm{TeV}$ (again depending on its contribution).
\end{abstract}

\section{Motivations and notation}

Precision studies of the interaction vertices between the top quark and the gauge bosons provide an important tool in testing the standard model (SM) against new-physics contributions. Currently available measurements from the Tevatron and the LHC already allow one to set stringent limits on possible deviations in the values of these couplings from their SM values. A model independent framework to study these anomalous couplings is provided by effective field theory, where the modifications are encoded into the coefficients of a set of higher dimensional operators that parametrize the effects of new physics at low energy.

The top quark has both strong and electroweak (EW) interactions. While all interactions come together in collider physics, it is possible to separate in most processes the EW from the strong part so that the anomalous vertices can be discussed separately. In this paper we concentrate on the

\footnotetext{
a e-mail: marco@sissa.it

b e-mail: michele.pinamonti@gmail.com

c e-mails: alberto.tonero@gmail.com; tonero@ift.unesp.br
}

study of the $W t b$ vertex; the strong interaction $G t \bar{t}$ vertex was recently studied in [1] — the results of which are here reported. Possible deviations in the interaction between the top quark and the neutral bosons $Z$ and $\gamma$-they enter the associated productions $t \bar{t} Z$ and $t \bar{t} \gamma$-are left out because they still are poorly measured.

\subsection{Effective $W t b$ vertex}

Following the literature [2], we write the effective lagrangian that describes, after EW symmetry breaking, the most general $W t b$ vertex as

$$
\begin{aligned}
\mathcal{L}_{W t b}= & -\frac{g}{\sqrt{2}} \bar{b} \gamma^{\mu}\left(V_{L} P_{L}+V_{R} P_{R}\right) t W_{\mu}^{-}-\frac{g}{\sqrt{2}} \bar{b} \frac{i \sigma^{\mu v} q_{\nu}}{m_{W}} \\
& \times\left(g_{L} P_{L}+g_{R} P_{R}\right) t W_{\mu}^{-}+\text {h.c. },
\end{aligned}
$$

where $g$ is the $S U(2)_{L}$ gauge coupling, $P_{L, R}$ the chiral projectors are $\left(1 \pm \gamma_{5}\right) / 2$, and $\sigma^{\mu \nu}=i\left[\gamma^{\mu}, \gamma^{\nu}\right] / 2$. The coefficients $V_{L}, V_{R}, g_{L}$, and $g_{R}$ are, in general, complex dimensionless constants. In this work we will restrict ourselves to the CP-conserving case and these couplings are taken to be real. In the SM the $W t b$ vertex in Eq. (1) reduces at the tree level to the Dirac vertex with $V_{L}=1$ (after mass diagonalization, $V_{L}=V_{t b} \simeq 1$ ) and we take it to be positive. Corrections to $V_{L}$, as well as the other non-zero anomalous couplings $V_{R}, g_{L}$, and $g_{R}$ can be generated by new physics.

If the physics beyond the SM lies at an energy scale $\Lambda$ that is larger than the EW scale $v$, then we can parametrize its effects via higher dimensional operators respecting the SM symmetries in a series suppressed by inverse powers of the scale $\Lambda$. The leading contributions arise at dimension six and can be written as an expansion of the local operators $\left\{\hat{O}_{k}\right\}$,

$\mathcal{L}_{\mathrm{SM}}^{\operatorname{dim} 6}=\sum_{k} \frac{c_{k}}{\Lambda^{2}} \hat{O}_{k}$

where $c_{k}$ are dimensionless coefficients. 
The complete list of independent dimension six SM effective operators is reported in [3] of which we follow the notation. The subset of operators that contributes to the anomalous couplings in Eq. (1) consists of the following operators:

$$
\begin{aligned}
& \hat{O}_{\varphi q}^{(3)}=\left(\varphi^{\dagger} i \overleftrightarrow{D}_{\mu}^{I} \varphi\right)\left(\bar{q}_{L} \sigma^{I} \gamma^{\mu} q_{L}\right) \\
& \hat{O}_{\varphi t b}=i\left(\tilde{\varphi}^{\dagger} D_{\mu} \varphi\right)\left(\bar{t}_{R} \gamma^{\mu} b_{R}\right) \\
& \hat{O}_{t W}=\bar{q}_{L} \sigma^{\mu \nu} \sigma^{I} t_{R} \tilde{\varphi} W_{\mu \nu}^{I}, \\
& \hat{O}_{b W}=\bar{q}_{L} \sigma^{\mu \nu} \sigma^{I} b_{R} \varphi W_{\mu \nu}^{I}
\end{aligned}
$$

where $q^{T}=(t, b), \tilde{\varphi}=i \sigma^{2} \varphi^{*}$, and $D_{\mu} \varphi=\partial_{\mu} \varphi+$ $i g W_{\mu}^{I} \frac{\sigma^{I}}{2} \varphi+i g^{\prime} Y_{\varphi} B_{\mu} \varphi$. After EW symmetry breaking $\varphi=$ $(0, v+H / \sqrt{2}), v=246 \mathrm{GeV}$, we can express the anomalous couplings in terms of the effective field theory coefficients as follows:

$$
\begin{aligned}
V_{L} & =V_{t b}+c_{\varphi q}^{(3)} \frac{v^{2}}{\Lambda^{2}} \simeq 1+c_{\varphi q}^{(3)} \frac{v^{2}}{\Lambda^{2}} \\
V_{R} & =\frac{1}{2} c_{\varphi t b} \frac{v^{2}}{\Lambda^{2}} \\
g_{L} & =\sqrt{2} c_{b W} \frac{v^{2}}{\Lambda^{2}} \\
g_{R} & =\sqrt{2} c_{t} \frac{v^{2}}{\Lambda^{2}} .
\end{aligned}
$$

There is another operator that enters in the processes we consider: the four-fermion operator

$\hat{O}_{q q^{\prime}}^{(3)}=\bar{q}_{L} \gamma^{\mu} \sigma^{I} q_{L} \bar{q}_{L}^{\prime} \gamma^{\mu} \sigma^{I} q_{L}^{\prime}$,

where $q^{\prime T}=(u, d)$ and, as before, $q^{T}=(t, b)$. It does not give a direct contribution to the anomalous couplings but its interplay with the other operators modifies the limits. For this reason it must be included and its effect parametrized by the new coefficient

$C_{4 f}=c_{q q^{\prime}}^{(3)} \frac{v^{2}}{\Lambda^{2}}$

which can be further identified by taking $c_{q q^{\prime}}^{(3)}=2 \pi$ with the usual convention of writing four-quark operators as

$\frac{2 \pi}{\Lambda^{2}} \bar{\psi}_{L} \gamma^{\mu} \psi_{L} \bar{\psi}_{L} \gamma_{\mu} \psi_{L}$

where the coefficient $2 \pi$ represents the strength of a strongly interacting new-physics sector-the integration of which gives rise to the effective operator.

The effect of these operators on the top-quark EW anomalous couplings can be best studied in two processes: top decay (by means of the $W$ polarizations) and single-top production.

\subsection{Effective $G t \bar{t}$ vertex}

The effective vertex for the $G t \bar{t}$ interactions can be written as

$\mathcal{L}_{G t \bar{t}}=-g_{s} \bar{t}\left[\gamma^{\mu} F_{1}\left(q^{2}\right)+\frac{i \sigma^{\mu v} q_{v}}{2 m_{t}} F_{2}\left(q^{2}\right)\right] G_{\mu} t$,

where $g_{s}$ is the strong $S U(3)_{C}$ coupling constant, $G_{\mu}=$ $T_{A} G_{\mu}^{A}$ is the gluon field, $T_{A}$ are the $S U(3)_{C}$ group generators, $q^{\mu}$ is the momentum carried by the gluon, and $t$ denotes the top-quark field. The interaction in Eq. (8) is the most general one on assuming that the vector-like nature of the gluon-top quark vertex is preserved by the underlying dynamics giving rise to the composite state.

The leading contributions come from the following two higher dimensional operators:

$$
\begin{gathered}
\hat{O}_{1}=g_{s} \frac{C_{1}}{m_{t}^{2}} \bar{t} \gamma^{\mu} T_{A} t D^{\nu} G_{\mu \nu}^{A} \text { and } \\
\hat{O}_{2}=g_{s} \frac{C_{2} v}{2 \sqrt{2} m_{t}^{2}} \bar{t} \sigma^{\mu \nu} T_{A} t G_{\mu \nu}^{A}
\end{gathered}
$$

where $D^{\nu} G_{\mu \nu}^{A}=\partial^{\nu} G_{\mu \nu}^{A}+g_{s} f^{A}{ }_{B C} G^{\nu B} G_{\mu \nu}^{C}, G_{\mu \nu}^{A}=$ $\partial_{\mu} G_{v}^{A}-\partial_{\nu} G_{\mu}^{A}+g_{s} f^{A}{ }_{B C} G_{\mu}^{B} G_{v}^{C}$ is the gluon field strength tensor, and the $f^{A}{ }_{B C}$ are the $S U(3)_{C}$ structure constants. In Eq. (9) the operator $\hat{O}_{1}$ and $\hat{O}_{2}$ are, respectively, of dimension six and five. We limit ourselves to the $C P$-conserving case and the dimensionless coefficients $C_{1}$ and $C_{2}$ are taken to be real. The operator $\hat{O}_{1}$ gives the leading $q^{2}$ dependence to $F_{1}$, while $\hat{O}_{2}$ gives the $q^{2}$-independent term of $F_{2}$ :

$F_{1}\left(q^{2}\right)=1+C_{1} \frac{q^{2}}{m_{t}^{2}}+\cdots \quad$ and $\quad F_{2}(0)=\sqrt{2} C_{2} \frac{v}{m_{t}}$.

Operators of higher dimensions can in general contributethey gives further terms in the expansion of the form factorsbut their effect is very much suppressed. The form of the coefficients in front of the operators in Eq. (9) is conventional and dictated, in our case, by the analogy with the electromagnetic form factors. In addition, the operator $\hat{O}_{2}$ is written for convenience with an extra factor $v / m_{t}$ because it can be thought of as coming, after EW symmetry breaking, from a dimension six $S U(3)_{C} \times S U(2)_{L} \times U(1)_{Y}$ gauge invariant operator that includes the Higgs boson field.

These vertices were analyzed (most recently in [1] where references to all related works can be found) by means of the $t \bar{t}$ production cross section and spin correlations. The following limits are found:

$-0.008 \leq C_{1} \leq 0.015$ and $-0.023 \leq C_{2} \leq 0.020$. 
These limits give a direct bound on the magnetic moment $\mu$ and the RMS radius $\left\langle r^{2}\right\rangle$, traditionally used to characterize the size of extended objects. They correspond to

$$
\begin{aligned}
& \sqrt{\left\langle r^{2}\right\rangle}<4.6 \times 10^{-4} \mathrm{fm} \quad(95 \% \mathrm{CL}) \\
& \text { and }-0.046<\mu-1<0.040 \quad(95 \% \mathrm{CL}) .
\end{aligned}
$$

We take the coefficients $C_{1}$ and $C_{2}$ to be zero when studying EW anomalous couplings-they only enter one channel of the single-top production-and assume that the strong interaction of the top quark follows the SM in all production processes.

\section{Methods}

In order to study the effect of anomalous couplings on top decay rates and single-top production cross sections at the LHC and Tevatron, we first use FEYNRULES [4] to implement our model, which has been defined to be the SM with the addition of the effective operators in Eqs. (3) and (5). The dependence on these operators is encoded in the coefficients $c_{i}=V_{L}, V_{R}, g_{L}, g_{R}, C_{4 f}$.

FEYNRULES provides the Universal FeynRules Output (UFO) with the Feynman rules of the model. The UFO is then used by MADGRAPH 5 [5] (MG5) to compute the branching ratios in the decay rates and production cross sections, which we denote by $F_{L, 0}^{\mathrm{MG} 5}$ and $\sigma_{\mathrm{MG} 5}$, respectively.

MG5 computes the square of the amplitude for each single channel. The partonic level result thus obtained can be compared with the partonic experimental cross section that is extracted by the experimental collaborations.

We compute, using MG5, the top-quark decay width and the single-top production cross section varying the values of the anomalous couplings $c_{i}$ in a range that goes from -2 to 2 -except for $V_{L}$ that is varied only for positive values. These different values of branching ratios $F_{L, 0}^{\mathrm{MG} 5}\left(c_{i}\right)$ and cross sections $\sigma_{\mathrm{MG} 5}\left(c_{i}\right)$ are used to obtain the limits on the coefficients by comparing the MG5 computation with the measured cross sections and helicity fractions at the LHC at the center-ofmass (CM) energies $\sqrt{s}=7$ and 8 , as discussed in the next section. By proceeding in the same way, we have also computed the rate and production cross section at the Tevatron and compared it with the measured cross section at the $\mathrm{CM}$ energy $\sqrt{s}=1.98 \mathrm{TeV}$.

Analytical expressions for the amplitudes that we study numerically can be found in [6].

\subsection{Statistical analysis}

To obtain $95 \%$ confidence level (CL) limits on the coefficients $c_{i}$ from the production cross sections, we use the quantity $\Delta \sigma_{\exp }$, defined to be the difference between the central value of the measured cross section $\bar{\sigma}_{\exp }$ and the theoretical value of the SM, $\bar{\sigma}_{\mathrm{th}}$ :

$\Delta \sigma_{\text {exp }}=\bar{\sigma}_{\text {exp }}-\bar{\sigma}_{\text {th }}$.

The uncertainty on $\Delta \sigma_{\exp }$ is given by summing in quadrature the respective uncertainties:

$\sqrt{\left(\delta \sigma_{\text {exp }}\right)^{2}+\left(\delta \sigma_{\mathrm{th}}\right)^{2}}$.

Using the cross sections $\sigma_{\mathrm{MG} 5}\left(c_{i}\right)$ calculated with MG5 we compute the value of the cross section coming from new physics as:

$\Delta \sigma_{\mathrm{MG} 5}\left(c_{i}\right)=\sigma_{\mathrm{MG} 5}\left(c_{i}\right)-\sigma_{\mathrm{MG} 5}(0)$.

The quantity $\Delta \sigma_{\mathrm{MG} 5}\left(c_{i}\right)$ represents the contribution to the cross section coming from the interference between SM leading order and new-physics diagrams as well as terms quadratic in the anomalous couplings.

Values of $c_{i}$ for which $\Delta \sigma_{\mathrm{MG} 5}\left(c_{i}\right)$ is more than two standard deviations from $\Delta \sigma_{\text {exp }}$, namely

$\Delta \sigma_{\mathrm{MG} 5}\left(c_{i}\right)>\Delta \sigma_{\mathrm{exp}}+2 \sqrt{\left(\delta \sigma_{\mathrm{exp}}\right)^{2}+\left(\delta \sigma_{\mathrm{th}}\right)^{2}}$

or

$\Delta \sigma_{\mathrm{MG} 5}\left(c_{i}\right)<\Delta \sigma_{\mathrm{exp}}-2 \sqrt{\left(\delta \sigma_{\mathrm{exp}}\right)^{2}+\left(\delta \sigma_{\mathrm{th}}\right)^{2}}$,

are considered excluded at $95 \% \mathrm{CL}$.

To obtain the limits from the branching ratios in top decay, as measured by the $W$-boson helicity fractions, a similar procedure is used. The experiments measure the three fractions $F_{0}, F_{L}$, and $F_{R}$ (see below for the definition). Being extracted from the same data set and from the same observable, these fractions are not independent. $F_{R}$ is constrained to be equal to $1-F_{0}-F_{L}$ and is therefore not considered in the limit extraction. In addition, there is a correlation factor $\rho$ different from 0 between the measured values of $F_{0}$ and $F_{L}$.

The helicity fractions $F_{0}^{\mathrm{MG} 5}\left(c_{i}\right)$ and $F_{L}^{\mathrm{MG} 5}\left(c_{i}\right)$ are computed within MG5, after adjusting for higher order QCD corrections, and compared to the measured values $F_{0}^{\exp }$ and $F_{L}^{\exp }$. The only uncertainties we consider are the experimental uncertainties $\delta F_{0}^{\exp }$ and $\delta F_{L}^{\exp }$, the theoretical ones being negligible.

To extract the limits, a likelihood function is defined as:

$L\left(c_{i}\right) \propto e^{-\frac{1}{2\left(1-\rho^{2}\right)}\left[\chi_{0}^{2}\left(c_{i}\right)+\chi_{L}^{2}\left(c_{i}\right)-2 \rho \cdot \chi_{0}\left(c_{i}\right) \cdot \chi_{L}\left(c_{i}\right)\right]}$,

where

$\chi_{0}\left(c_{i}\right)=\frac{F_{0}^{\exp }-F_{0}^{\mathrm{MG} 5}\left(c_{i}\right)}{\delta F_{0}}$ and $\chi_{L}\left(c_{i}\right)=\frac{F_{L}^{\exp }-F_{L}^{\mathrm{MG} 5}\left(c_{i}\right)}{\delta F_{L}}$,

and the constraint $\int L\left(c_{i}\right) d c_{i}=1$ is imposed. 
Then the quantity $L^{95 \%}$ is defined as

$\int_{L\left(c_{i}\right)>L^{95 \%}} L\left(c_{i}\right) d c_{i}=0.95$

and values of $c_{i}$ for which $L\left(c_{i}\right)>L^{95 \%}$ are considered excluded at $95 \%$ CL.

\section{Results}

Top-quark decay and single-top production are the two processes where we can directly probe the top coupling to the weak bosons.

The Wbt vertex in top-quark decay is best studied by means of the helicity fractions $F_{0}$ and $F_{L}$ defined, respectively, as the ratios between the rates of polarized decay of the top quark into zero and left-handed $W$ bosons and the total decay width. In the SM they are found, neglecting $m_{b}$, to be $F_{0}=\frac{m_{t}^{2}}{m_{t}^{2}+2 m_{W}^{2}} \simeq 0.7$ and $F_{L}=\frac{2 m_{W}^{2}}{m_{t}^{2}+2 m_{W}^{2}} \simeq 0.3 ;$

the helicity fraction into right-handed $W$ bosons is vanishingly small. These branching ratios receive corrections from the operators $\hat{O}_{t W}$ and $\hat{O}_{b W}$. The SM values at the next-tonext-to-leading order (NNLO) order in QCD are computed in $[7,8]$.

The best current experimental results are given by CMS [9] for data at $\sqrt{s}=8 \mathrm{TeV}$ (integrated luminosity $19.6 \mathrm{fb}^{-1}$ ) as

$F_{0}=0.659 \pm 0.015$ (stat.) \pm 0.023 (syst.)

$F_{L}=0.350 \pm 0.010$ (stat.) \pm 0.024 (syst.),

with a correlation coefficient $\rho=0.95$.

The single-top production occurs through $t$-, $s$ - and $W t$ channel (see Fig. 1). The SM NNLO cross sections have been computed in [10-12], respectively. The corresponding experimental cross sections $\sigma_{t}, \sigma_{s}$ and $\sigma_{W t}$ are available from the LHC and Tevatron data and we utilize the following results (in which the error includes both statistical and systematic contributions):

$\sigma_{t}=67.2 \pm 6.1 \mathrm{pb} \quad(\mathrm{LHC} @ 7 \mathrm{TeV})[13]$

$\sigma_{t}=83.6 \pm 7.7 \mathrm{pb} \quad(\mathrm{LHC} @ 8 \mathrm{TeV})[14]$,

for single-top production in the $t$-channel (integrated luminosities of 1.17 and $1.56 \mathrm{fb}^{-1}$ for, respectively, muon and

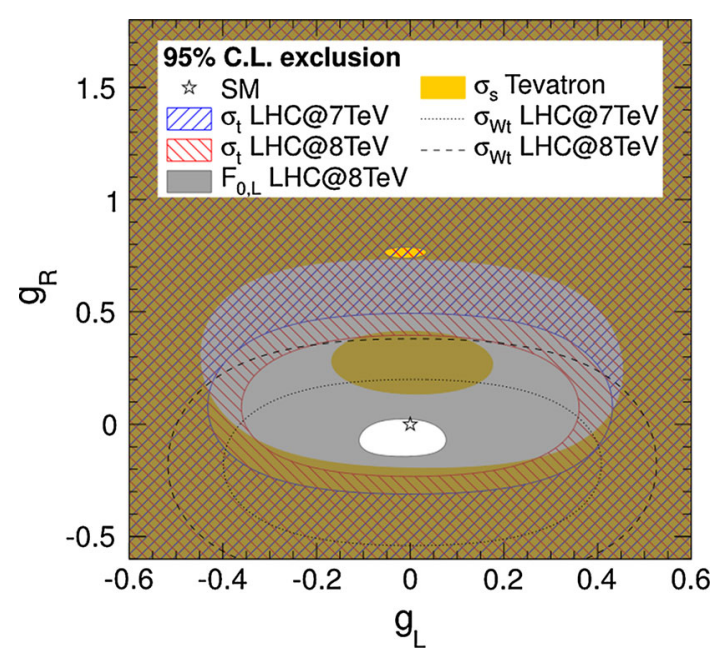

Fig. $295 \%$ CL exclusion limits on the coefficients $g_{R}$ and $g_{L}\left(V_{L}=1\right.$, $\left.V_{R}=C_{4 f}=0\right)$. The full yellow, striped red, striped blue, and shaded gray areas indicate the excluded regions from the $s$ - and $t$-channel production cross sections and from the helicity fractions. Brown areas result from the superposition of yellow and gray areas. The area outside the dashed (dotted) ellipses is excluded by the $W t$-channel cross section measurement at the $\mathrm{LHC}$ at $8 \mathrm{TeV}(7 \mathrm{TeV})$. The region of allowed values is $-0.109 \leq g_{L} \leq 0.076$ and $-0.142 \leq g_{R} \leq 0.024$

electron final states at $7 \mathrm{TeV}$ and $19.7 \mathrm{fb}^{-1}$ at $8 \mathrm{TeV}$ ),

$\sigma_{s}=1.29_{-0.24}^{+0.26}(\mathrm{CDF}+\mathrm{D} 0 @ 1.98)[15]$,

for the $s$-channel (integrated luminosity $7.5 \mathrm{pb}^{-1}$ ) and

$\sigma_{W t}=16_{-4}^{+5} \mathrm{pb} \quad(\mathrm{LHC} @ 7 \mathrm{TeV})[16]$

$\sigma_{W t}=25 \pm 4.7 \mathrm{pb} \quad(\mathrm{LHC} @ 8 \mathrm{TeV})[17]$,

for the $W t$-channel (integrated luminosities $4.9 \mathrm{fb}^{-1}$ at $7 \mathrm{TeV}$ and $12.2 \mathrm{fb}^{-1}$ at $8 \mathrm{TeV}$ ).

We study the effect of the operators in Eqs. (3) and (5) by varying the coefficients of two of them at the time and we derive the related limits following the statistical analysis described in the previous section. The results are shown in Figs. 2, 3, and 4 .

The determination of the limits on the coefficients $g_{R}$ and $g_{L}$ is dominated by the top-quark decay but the single-top production cross section is useful in eliminating larger values (the small area on top of Fig. 2). Accordingly, only the lower region is allowed and

$$
-0.109 \leq g_{L} \leq 0.076 \text { and }-0.142 \leq g_{R} \leq 0.024 \text {. }
$$

Fig. 1 Tree-level diagrams for, from left to right, $s$-, $t$ - and $W t$-channel in top-quark production

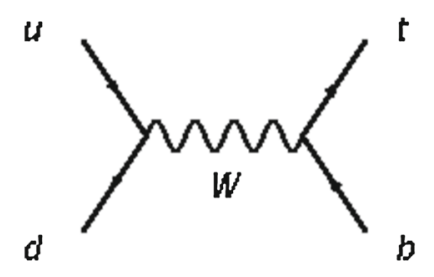

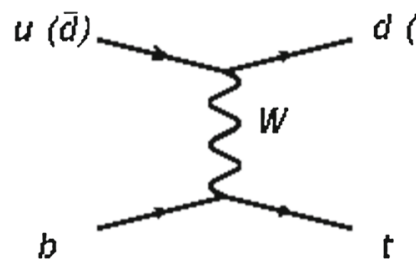

$d(\bar{u})$

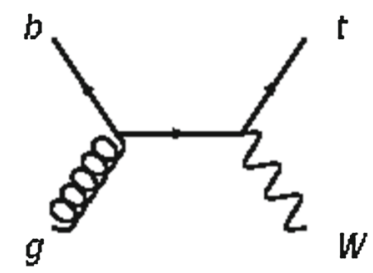




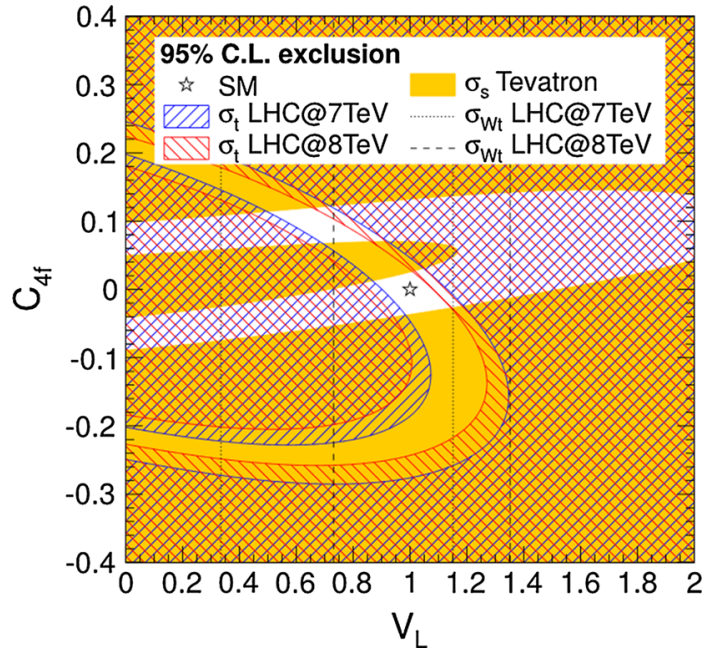

Fig. $395 \% \mathrm{CL}$ exclusion limits on the coefficients $C_{4 f}$ and $V_{L}$ $\left(g_{L}=g_{R}=V_{R}=0\right)$. The full yellow, striped red, striped blue, and shaded gray areas indicate the excluded regions from the $s$ - and $t$-channel production cross sections and from the helicity fractions. The areas outside the dashed (dotted) vertical lines are excluded by the $W t$-channel cross-section measurement at the LHC at $8 \mathrm{TeV}$ (7 $\mathrm{TeV}$ ). The region of allowed values is $0.732 \leq V_{L} \leq 1.145$ and $-0.037 \leq C_{4 f} \leq 0.120$

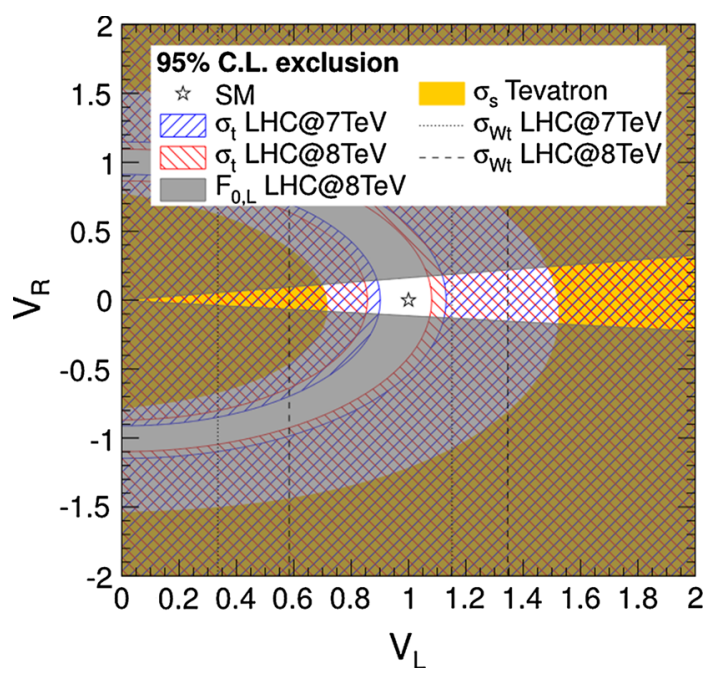

Fig. $495 \%$ CL exclusion limits on the coefficients $V_{R}$ and $V_{L}\left(g_{L}=\right.$ $\left.g_{R}=C_{4 f}=0\right)$. The full yellow, striped red, striped blue, and shaded gray areas indicate the excluded regions from the $s$ - and $t$-channel production cross sections and from the helicity fractions. Brown areas result from the superposition of yellow and gray areas. The areas outside the dashed (dotted) vertical lines are excluded by the $W t$-channel crosssection measurement at the $\mathrm{LHC}$ at $8 \mathrm{TeV}(7 \mathrm{TeV})$. The region of allowed values is $0.891 \leq V_{L} \leq 1.081$ and $-0.121 \leq V_{R} \leq 0.173$

The interplay among the various channels of single-top production is crucial in delimiting the allowed region for the parameters $C_{4 f}$ and $V_{L}$ in Fig 3. Unfortunately, the $W t$ channel is not sensitive to $V_{R}$ and $C_{4 f}$ and the remaining channels are not sufficient in completely delimiting the allowed range of the coefficients to a single region. Accord-
Table 1 Limits $(95 \% \mathrm{CL})$ on the coefficients $c_{i}=V_{L}, V_{R}, g_{L}$, $g_{R}, C_{4 f}$, and $C_{1,2}$ when they are varied independently of each other and energy scale of the corresponding effective operators

\begin{tabular}{ll}
\hline$-0.142 \leq g_{R} \leq 0.023$ & $\left|g_{R}\right| \leq 0.142, \Lambda \gtrsim 780 \mathrm{GeV}$ \\
$-0.081 \leq g_{L} \leq 0.049$ & $\left|g_{L}\right| \leq 0.081, \Lambda \gtrsim 1 \mathrm{TeV}$ \\
$0.902 \leq V_{L} \leq 1.081$ & $\left|V_{L}-1\right| \leq 0.0099, \Lambda \gtrsim 780 \mathrm{GeV}$ \\
$-0.112 \leq V_{R} \leq 0.162$ & $\left|V_{R}\right| \leq 0.162, \Lambda \gtrsim 430 \mathrm{GeV}$ \\
$-0.036 \leq C_{4} \leq 0.025$ & $\left|C_{4 f}\right| \leq 0.036, \Lambda \gtrsim 3.2 \mathrm{TeV}$ \\
$-0.008 \leq C_{1} \leq 0.015$ & $\left|C_{1}\right| \leq 0.015, \Lambda \gtrsim 1.3 \mathrm{TeV}$ \\
$-0.023 \leq C_{2} \leq 0.020$ & $\left|C_{2}\right| \leq 0.023, \Lambda \gtrsim 1.5 \mathrm{TeV}$ \\
\hline
\end{tabular}

ingly, the final bound is weakened when both coefficients are allowed to vary simultaneously and we find

$0.732 \leq V_{L} \leq 1.145$ and $-0.037 \leq C_{4 f} \leq 0.120$.

A future improvement in the $W t$-channel measurement at the LHC could be instrumental in delimiting the range to a single region.

Finally, it is the interplay between top decay and the $t$ channel of single-top production that provides the limit on the coefficients $V_{R}$ and $V_{L}$ (see Fig. 4). We find

$0.891 \leq V_{L} \leq 1.081$ and $-0.121 \leq V_{R} \leq 0.173$.

Notice that all these coefficient are very much constrained by flavor physics. In particular, the coefficient $V_{R}$ should be [13]

$-0.0004 \leq V_{R} \leq 0.0013$.

When this limit is included, Fig. 4 should be read only along the line $V_{R} \simeq 0$ with a slightly improved bound

$0.902 \leq V_{L} \leq 1.081$.

Similarly, the coefficient $g_{L}$ should be approximately less than $0.001[14,15]$. Limits comparable to those from collider physics hold for the other coefficients.

Our results are summarized in Table 1 in the case in which the various anomalous couplings are turned on one at the time.

The limits found can be interpreted in terms of the energy scale of the effective operators in Eq. (3) (see second column of Table 1). By inspection, we see that all EW limits are around $10^{-1}$ which translates into a characteristic scale $\Lambda \simeq$ $700 \mathrm{GeV}$ (actually from $430 \mathrm{GeV}$ to $1 \mathrm{TeV}$, depending on the contribution) except for the four-quark interaction which has $\Lambda \simeq 3.2 \mathrm{TeV}$, if we follow the definition in Eq. (6). Above these limits, there is still room for new physics. These results can be compared with the strong sector interactions of the top quark where the corresponding energy scale is $\Lambda \simeq 1.3$ and $1.5 \mathrm{TeV}$ for, respectively, the operators $\hat{O}_{1}$ and $\hat{O}_{2}$ in Eq. (9) [1]. 

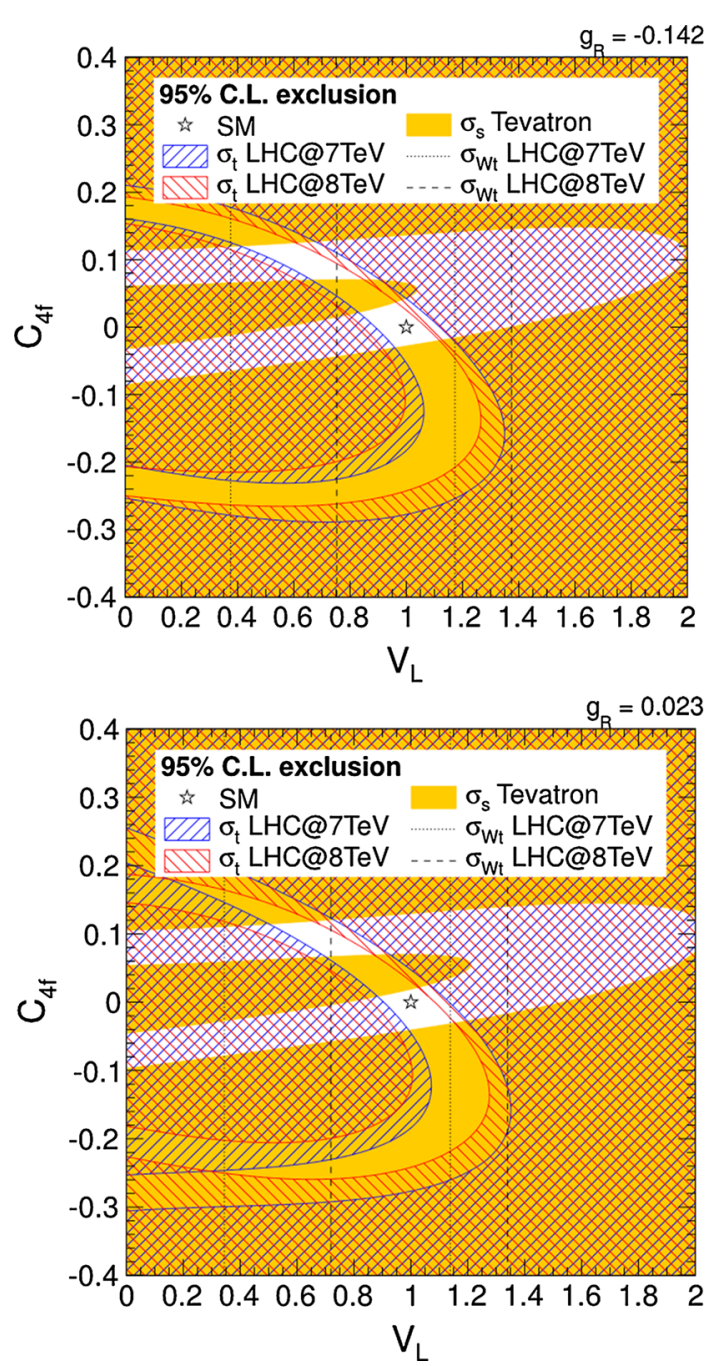

Fig. $595 \% \mathrm{CL}$ exclusion limits on the coefficients $C_{4 f}$ and $V_{L}$ $\left(g_{L}=V_{R}=0\right)$ for the two extreme allowed values of the coefficient $g_{R}=-0.142$ (top) and $g_{R}=-0.023$ (bottom). The full yellow, striped red, striped blue, and shaded gray areas indicate the excluded regions from the $s$ - and $t$-channel production cross sections and from the helicity fractions. The areas outside the dashed (dotted) vertical lines are excluded by the $W t$-channel cross-section measurement at the $\mathrm{LHC}$ at $8 \mathrm{TeV}(7 \mathrm{TeV})$

\section{Discussion}

Constraints on the $W t b$ vertex from Tevatron and early LHC data were reported in [16]. Future limits for the LHC at 14 $\mathrm{TeV}$ were estimated in [17]. The bounds were obtained, as in this paper, by combining the experimental measurements of $W$-boson helicity fractions and single-top production and by varying two operators at the time. More recently, the measurement of the $W$-boson helicity fractions has been substantially improved by ATLAS and CMS in [18-20] and limits on the coefficients $g_{R}$ and $g_{L}$ were extracted by the experimental collaborations based on these new measurements. Concerning the single-top production, many detailed results have been released by both Tevatron and LHC experiments [2125], as reported in the previous section. The improvement is substantial, in most cases of almost one order of magnitude.

We confirm and improve the above limits by means of the most recent data set at $8 \mathrm{TeV}$ by CMS [9] and the combined use of the helicity fractions and single-top production cross sections. The result for the four-fermion operator (5), and its impact on the determination of the coefficient $V_{L}$ is new.

As expected, the simultaneous presence of more than one anomalous coupling weakens the limits. We have verified that the variation of more than two of the coefficients does not significantly change the result. As an example, in Fig. 5, we show how the limits on the coefficients $V_{L}$ vs. $C_{4 f}$ are changed by taking the two extreme values among those allowed for the coefficient $g_{R}$. For $g_{R}=-0.142$ we have

$0.753<V_{L}<1.116$ and $-0.030<C_{4 f}<0.094$,

while for $g_{R}=0.023$

$0.720<V_{L}<1.138$ and $-0.041<C_{4 f}<0.105$.

These results show that the variation in the value of the limits is around $20 \%$. Simultaneous variation of other subsets of coefficients, with respect to their limits as taken two at the time, are even smaller. This is explained by the dominance of different processes in the constraints for different pairs of coefficients: helicity fractions in top-quark decay dominate the limits for $g_{L}$ and $g_{R}$ while single-top production dominates those of $V_{L}$ and $C_{4 f}$.

Acknowledgments MF is associated to SISSA. The work of AT was supported by the São Paulo Research Foundation (FAPESP) under grants 2011/11973-4 and 2013/02404-1.

Open Access This article is distributed under the terms of the Creative Commons Attribution License which permits any use, distribution, and reproduction in any medium, provided the original author(s) and the source are credited.

Funded by $\mathrm{SCOAP}^{3}$ / License Version CC BY 4.0.

\section{References}

1. M. Fabbrichesi, M. Pinamonti, A. Tonero, Stringent limits on topquark compositeness from top anti-top production at the Tevatron and the LHC. Phys. Rev. D 89, 074028 (2014). arXiv: 1307.5750 [hep-ph]

2. J.A. Aguilar-Saavedra, A minimal set of top anomalous couplings. Nucl. Phys. B 812, 181 (2009). arXiv:0811.3842 [hep-ph]

3. B. Grzadkowski, M. Iskrzynski, M. Misiak, J. Rosiek, Dimensionsix terms in the standard model Lagrangian. JHEP 1010, 085 (2010). arXiv:1008.4884 [hep-ph]

4. N.D. Christensen, C. Duhr, FeynRules-Feynman rules made easy. Comput. Phys. Commun. 180, 1614 (2009). arXiv:0806.4194 [hep$\mathrm{ph}]$

5. J. Alwall et al., MadGraph 5: going beyond. JHEP 1106, 128 (2011). arXiv:1106.0522 [hep-ph] 
6. C. Zhang, S. Willenbrock, Effective-field-theory approach to topquark production and decay. Phys. Rev. D 83, 034006 (2011). arXiv:1008.3869 [hep-ph]

7. J. Gao, C.S. Li, H.X. Zhu, Top quark decay at next-to-nextto leading order in QCD. Phys. Rev. Lett. 110, 042001 (2013). arXiv:1210.2808 [hep-ph]

8. A. Czarnecki, J.G. Korner, J.H. Piclum, Helicity fractions of W bosons from top quark decays at NNLO in QCD. Phys. Rev. D 81, 111503 (2010). arXiv:1005.2625 [hep-ph]

9. [CMS Collaboration], Measurement of the W-boson helicity in top decays from ttbar production in lepton+jets events at the LHC at $\operatorname{sqrt}(\mathrm{s})=8 \mathrm{TeV}$. CMS-PAS-TOP-13-008

10. N. Kidonakis, Next-to-next-to-leading-order collinear and soft gluon corrections for t-channel single top quark production. Phys. Rev. D 83, 091503 (2011). arXiv:1103.2792 [hep-ph]

11. N. Kidonakis, NNLL resummation for s-channel single top quark production. Phys. Rev. D 81, 054028 (2010). arXiv:1001.5034 [hep-ph]

12. N. Kidonakis, Next-to-next-to-leading soft-gluon corrections for the top quark cross section and transverse momentum distribution. Phys. Rev. D 82, 114030 (2010). arXiv:1009.4935 [hep-ph]

13. N. Vignaroli, $\Delta \mathrm{F}=1$ constraints on composite Higgs models with LR parity. Phys. Rev. D 86, 115011 (2012). arXiv:1204.0478 [hep$\mathrm{ph}]$

14. J. Drobnak, S. Fajfer, J.F. Kamenik, New physics in $t \rightarrow b W$ decay at next-to-leading order in QCD. Phys. Rev. D 82, 114008 (2010). arXiv:1010.2402 [hep-ph]

15. J. Drobnak, S. Fajfer, J.F. Kamenik, Probing anomalous tWb interactions with rare B decays. Nucl. Phys. B 855, 82 (2012). arXiv:1109.2357 [hep-ph]

16. J.A. Aguilar-Saavedra, N.F. Castro, A. Onofre, Constraints on the Wtb vertex from early LHC data. Phys. Rev. D 83, 117301 (2011). arXiv:1105.0117 [hep-ph]
17. F. Bach, T. Ohl, Anomalous top couplings at hadron colliders revisited. Phys. Rev. D 86, 114026 (2012). arXiv:1209.4564 [hep-ph]

18. [ATLAS Collaboration], Combination of the ATLAS and CMS measurements of the W-boson polarization in top-quark decays. ATLAS-CONF-2013-033

19. S. Chatrchyan et al., [CMS Collaboration], Measurement of the Wboson helicity in top-quark decays from $t \bar{t}$ production in lepton+jets events in pp collisions at $\sqrt{s}=7 \mathrm{TeV}$. arXiv:1308.3879 [hep-ex]

20. [CMS Collaboration], Measurement of W-helicity fractions in single top events topology. CMS-PAS-TOP-12-020

21. S. Chatrchyan et al., [CMS Collaboration], Measurement of the single-top-quark $t$-channel cross section in $p p$ collisions at $\sqrt{s}=7$ TeV. JHEP 1212, 035 (2012). arXiv: 1209.4533 [hep-ex]

22. V. Khachatryan et al., [CMS Collaboration], Measurement of the t-channel single-top-quark production cross section and of the $\left|V_{t b}\right|$ CKM matrix element in pp collisions at $\sqrt{s}=8 \mathrm{TeV}$. arXiv: 1403.7366 [hep-ex]

23. T.A. Aaltonen et al., [CDF and D0 Collaborations], Observation of s-channel production of single top quarks at the tevatron. arXiv: 1402.5126 [hep-ex]

24. S. Chatrchyan et al., [CMS Collaboration], Evidence for associated production of a single top quark and $\mathrm{W}$ boson in pp collisions at $\sqrt{s}=7$ TeV. Phys. Rev. Lett. 110, 022003 (2013). arXiv:1209.3489 [hep-ex]

25. [ATLAS and CMS collaborations], Combination of cross-section measurements for associated production of a single top-quark and a W boson at $\sqrt{s}=8 \mathrm{TeV}$ with the ATLAS and CMS. ATLASCONF-2014-052 and CMS-PAS-TOP-14-009 